\title{
Labyrinthe
}

29 | 2008 (1)

Ce que le libéralisme promet

\section{From passions to emotions}

The Creation of a Secular Psychological Category de Thomas Dixon

\section{Élodie Cassan}

\section{(2) OpenEdition}

\section{Journals}

Édition électronique

URL : http://journals.openedition.org/labyrinthe/3523

DOI : $10.4000 /$ labyrinthe.3523

ISSN : 1950-6031

Éditeur

Hermann

\section{Édition imprimée}

Date de publication : 11 janvier 2008

Pagination : 147-151

ISBN : 9782952613163

Référence électronique

Élodie Cassan, «From passions to emotions », Labyrinthe [En ligne], 29 | 2008 (1), mis en ligne le 11 janvier 2008, consulté le 19 novembre 2020. URL : http://journals.openedition.org/labyrinthe/3523 DOI : https://doi.org/10.4000/labyrinthe.3523 


\title{
FROM PASSIONS TO EMOTIONS The Creation \\ of a Secular Psychological Category de Thomas Dixon*
}

\author{
Élodie CASSAN \\ ecassan@gmail.com
}

Dans sa thèse de doctorat, Thomas Dixon fait le constat suivant : depuis une trentaine d'années, les études sur les émotions se développent, dans des domaines aussi variés que la psychologie cognitive, l'anthropologie et l'histoire littéraire. Par ailleurs, «l'industrie des émotions» ne se limite pas au monde académique. C'est ainsi par exemple qu'après le quotient intellectuel, il est désormais possible de calculer le quotient émotionnel.

À partir de là, Dixon se propose d'interroger cette reconnaissance et cette forte présence des émotions. À cette fin, il questionne une lecture de l'histoire de la philosophie morale en vogue dans la philosophie analytique. Selon celle-ci, les philosophes rationalistes occidentaux en général, et certains théologiens chrétiens en particulier, ont toujours conçu les passions (passions), ou émotions (emotions), termes synonymes à leurs yeux, en opposition à la raison (reason) : tyrans déréglés conduisant à mal agir malgré soi, les émotions doivent être réprimées par la raison et la volonté (will). La vision totalement négative dont les passions feraient l'objet dans la tradition rationaliste rendrait ainsi leur réhabilitation nécessaire. D'où, par exemple, l'œuvre de Robert Solomon ${ }^{1}$. Contre une approche «classique» des passions qui réduirait celles-ci à des phénomènes corporels, involontaires et irrationnels, Solomon s'efforce de montrer que les émotions sont en réalité des jugements cognitifs dont la formation requiert une activité

\footnotetext{
* Cambridge, Cambridge University Press, 2006 [1 $1^{\text {re }}$ éd. 2003], 287 p., $36 €$.

1. Robert Solomon, The Passions : Emotions and the Meaning of Life, Indianapolis, Hackett, 1976.
} 
de la volonté, et que, sans se réduire à ce que le corps fait subir, elles mettent en jeu l'interaction entre l'homme et le monde. Or, tout comme de nombreux et récents travaux sur la question des émotions, cette thèse repose sur un supposé antagonisme entre la raison et les passions. Il s'agit pour Dixon d'établir que ce présupposé relève plutôt du mythe que de la réalité textuelle, et par conséquent, que le projet moral des philosophies rationalistes ne saurait se ramener purement et simplement à une opposition entre la raison et les émotions, et à l'effort de la raison pour contrôler les émotions.

Dixon entreprend ainsi de reconstituer l'invention de la catégorie psychologique d'émotion. En montrant que cette création est beaucoup trop tardive pour que la catégorie d'émotion soit strictement superposable à celle de passion, il remet en question l'idée selon laquelle cette dernière couvrirait tout le champ de l'affectivité et, du même coup, la capacité théorique du couple raison-passion à rendre compte à lui seul de la sphère pratique.

Sa thèse consiste en effet à dire que la catégorie d'émotion naît au début du XIX ${ }^{e}$ siècle en Écosse, dans la philosophie empiriste de l'esprit. L'attribution d'un rôle cohérent, systématique et central au terme «émotion» en psychologie est l'œuvre des Lectures on the Philosophy of the Human Mind du philosophe écossais Thomas Brown, qui paraissent en 1820. Dans ce texte, Brown est influencé par le fait qu'en rejetant dans le Traité de la nature humaine une approche réaliste de l'esprit, c'est-à-dire, la thèse selon laquelle l'esprit est constitué de facultés qui s'expriment à travers des productions mentales, David Hume a pu considérer les passions non pas comme les actes d'une volonté individuelle mais comme des agents autonomes de l'esprit. Dans un cadre conceptuel baconien et newtonien, pour lequel l'étude de l'esprit humain passe par l'emprunt de méthodes et de concepts aux sciences de la matière, Brown se propose alors à la fois d'analyser la « chimie mentale ${ }^{2} », c^{\prime}$ 'est-à-dire d'identifier les composantes des états mentaux, et de découvrir les lois qui président à la succession de ces états. C'est dans cette perspective qu'il procède à une tripartition des phénomènes mentaux en sensations, pensées et émotions. Il accorde ainsi aux émotions le statut de catégorie psychologique. Celle-ci est

2. L'expression est de Brown. Elle a un sens analogique. Si Brown procède à des décompositions et à des divisions, comme en chimie, il tient un propos sur l'esprit qui ne relève pas de la physique. Il étudie les phénomènes mentaux en tant qu'ils sont mentaux. 
intermédiaire : participant d'une compréhension de l'action comme fruit de l'imposition de forces brutes à l'esprit, plutôt que de jugements raisonnés, les émotions ne sont pas des sensations, telles le goût ou l'odorat, et elles n'engagent pas non plus la cognition.

Mais instituer les émotions en lieu de l'affectivité ne va pas de soi. C'est faire fi des concepts de passion et d'affection (affection), à l'œuvre notamment chez Thomas d'Aquin, où ils désignent respectivement les mouvements de l'appétit sensible et ceux de la volonté. Or, si du point de vue de son extension, le concept d'émotion peut désigner différents sentiments qui relevaient au préalable des catégories d'appétit ou d'affection, en revanche, en intension, il diffère notablement du concept de passion. Alors que l'émotion n'est pas définie en termes moraux, mais simplement comme un état interne ressenti vivement et marqué par une agitation physique, la passion est communément appréhendée d'un point de vue moral et théologique comme un mouvement de l'âme désobéissant à la raison, voire comme un mouvement dangereux. Le changement lexical observé chez Brown recouvre donc un changement doctrinal. Celui-ci tient dans une prise de distance à l'égard d'une approche religieuse de l'âme. Il importe d'en dégager les conditions de possibilité et la portée. D'où la double orientation, historique et problématique, de l'ouvrage de Dixon.

Pour ce dernier, parler le langage des émotions et non plus celui des passions et des affections procède d'une sécularisation du discours psychologique, qui désigne ici, en un sens large, toute théorie sur la vie mentale, et n'est donc pas l'apanage de la psychologie scientifique moderne, physiologique, comportementale, neuronale, ou autre. Dixon montre que si les termes de «passion» et d' «affection» sont associés à ceux d' «âme», de «conscience», de «chute», de «péché», de «grâce », d' «Esprit», etc., celui d' «émotion» s'inscrit dans un réseau plus séculier de termes tels que ceux d' «observation », d' «évolution», d' «organisme», de «cerveau», de «nerfs», ou de «comportement». Par là, il ne veut pas à proprement parler identifier les théoriciens des passions et des affections à des penseurs chrétiens. Sa thèse consiste à dire, d'une part, que la distinction entre passions et affections, ainsi que ces catégories elles-mêmes, dérivent historiquement de psychologies d'inspiration théologique, et s'articulent à une compréhension christianisée de la personne humaine, en proie au péché et devant œuvrer pour son salut; pour Dixon, d'autre part, l'introduction du 
concept d'émotion, liée à la progressive constitution de la psychologie comme science, marque un écart par rapport à ce schéma théologique de l'esprit, sans pour autant impliquer sa remise en question.

Le passage des passions aux émotions comporte quatre étapes majeures. Tout d'abord, Dixon rend compte de la place et du statut des passions dans la philosophie morale chrétienne. Il rappelle qu'Augustin et Thomas d'Aquin distinguent en l'homme, outre une âme végétative, une âme rationnelle, qui se décompose en entendement et en volonté, et une âme sensible, irrationnelle. Il établit que si les passions relèvent de cette dernière, elles ne constituent pas le tout de l'affectivité. En effet, en sus des passions, celle-ci met également en jeu la volonté, en tant qu'elle a des affections. Elle a donc deux sources : des passions, désordres involontaires de l'âme liés à l'attrait d'objets matériels appréhendés par les sens, et des affections, mouvements volontaires de l'âme qui n'ont rien de moralement répréhensible, pour autant que la volonté soit bien conduite. Ensuite, préparée par une approche mécaniste des passions et des affections, la théorie brownienne des émotions unifie cette dualité sous le terme parapluie d'émotion, qui englobe ce qui n'est ni une sensation ni un état intellectuel. La catégorie brownienne d'émotion fait, dans un troisième temps, l'objet d'une appropriation par ces psychologues physicalistes, partisans de la théorie de l'évolution, que sont Herbert Spencer, Alexander Bain et Charles Darwin. La thèse d'inspiration chrétienne selon laquelle les passions et les affections sont des instances de l'âme qui ou bien agissent sur le corps, ou bien l'utilisent, est alors remplacée par l'idée que les émotions sont des instances du cerveau et des nerfs, qui agissent sur d'autres parties du corps. La théorie des émotions de William James, selon laquelle les changements corporels communément conçus comme l'expression d'une émotion en sont en réalité l'élément constituant, peut apparaître enfin, dans un quatrième et dernier temps, comme le résultat du développement de la psychologie réductionniste.

Cette reconstruction de l'invention de la catégorie psychologique d'émotion et de la façon dont elle en vient à recouvrir le champ traditionnellement attribué aux passions et aux affections est doublement instructive. D'une part, elle est porteuse d'une exigence de précision historique et conceptuelle. D'autre part, elle vaut par le regard critique qu'elle invite à jeter sur la philosophie analytique en tant que celle-ci se donne pour projet de mettre en lumière la rationalité des émotions. 
De nombreux auteurs travaillent à mettre en évidence une logique des émotions. Comment comprendre ce geste, sachant qu'à la différence des passions, les émotions ne se forment pas dans une opposition à la raison, et qu'elles ne constituent pas un objet limite qu'il faudrait apprendre à contrôler rationnellement? La lecture du livre de Dixon invite à se demander si en plus de procéder d'une confusion entre les concepts de passion et d'émotion, qui a été pointée, la détermination de cet objet de recherche ne traduit pas également un intérêt pour ce qui est supposé échapper à la rationalité, ainsi qu'un désir de promouvoir cet irrationnel. Elle mène alors à se demander si ce projet de réhabilitation ne témoigne pas d'une méfiance indue à l'égard de la raison, et de l'intelligence, sinon d'une approche réductrice de celles-ci comme facultés de l'esprit desséchantes et purement dogmatiques. From Passions to Emotions - The Creation of a Secular Psychological Category invite ainsi à réfléchir sur les fondements de la rationalité. 